\title{
Chondroitin Sulphate Proteoglycan is Involved in Lens Vesicle Morphogenesis in Chick Embryos
}

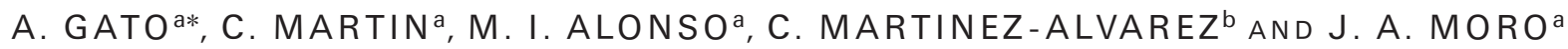 \\ ${ }^{a}$ Departamento de Anatomia Humana, Facultad de Medicina, Universidad de Valladolid, Spain and \\ ${ }^{\mathrm{b}}$ Departamento de Ciencias Morfológicas I, Facultad de Medicina, Universidad Complutense de Madrid, \\ Spain
}

(Received Oxford 9 January 2001 and accepted in revised form 13 June 2001)

\begin{abstract}
Proteoglycans have been implicated in the invagination and formation of various embryonal cavitied primordia. In this paper the expression of chondroitin sulphate proteoglycan is analysed (CSPG) in the lens primordium during lens vesicle formation, and demonstrate that this proteoglycan has a specific distribution pattern with regard to invagination and fusion processes in the transformation of placode into lens vesicle. More specifically, CSPG was detached in: (1) the apical surface of lens epithelial cells, where early CSPG expression was observed in the whole of the lens placode whilst in the vesicle phase it was restricted to the posterior epithelium; (2) intense CSPG expression in the basal lamina, which remained constant for the entire period under study; (3) CSPG expression in the intercellular spaces of the lens primordium epithelium, which increased during the invagination of the primordium and which at the vesicle stage was more evident in the posterior epithelium; and (4) CSPG expression on the edges of the lens placode both prior to and during fusion. Treatment with $\beta$-D-xyloside causes significant CSPG depletion in the lens primordium together with severe alterations in the invagination and fusion of the lens vesicle; this leads to the formation of lens primordia which in some cases remain practically flat or show partial invagination defects or fusion disruption. Similar results were obtained by enzyme digestion with chondroitinase AC but not with type II heparinase, which indicates that alterations induced by $\beta$-Dxyloside were due to interference in CSPG synthesis. The findings demonstrate that CSPG is a common component of the lens primordium at the earliest developmental stages during which it undergoes specific modifications. It also includes experimental evidence to show that 'in vivo' CSPG plays an important role

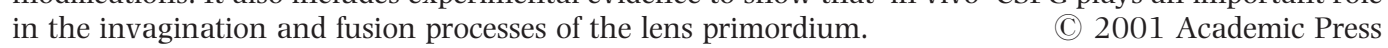

Key words: lens development; CSPG; $\beta$-D-xyloside; extracellular matrix; epithelial invagination.
\end{abstract}

\section{Introduction}

The earliest steps in lens development are based on a sequence of morphogenetic events which are common to other primordia and involve the induction of an ectodermal placode (lens placode) which invaginates and, subsequent to the approximation and fusion of its periferal limits, with the formation of the lens stalk and subsequent to its re-absorption, forms an isolated vesicle (lens vesicle). A morphological description of this process in chick embryos was given by Schook (1980a,b) and Hilfer (1983).

The morphogenetic processes involved in invagination leading to the formation of a tube or vesicle primordium are to a large extent unknown, although different mechanisms have been suggested; these include the following: (1) apical contraction of actin and myosin filaments which modulates the way in which the cellular cytoskeleton is organized and brings about changes in cellular shape. It has been suggested that this mechanism operates during the invagination of different ectodermic placodes such as

\footnotetext{
* Address correspondence to: Angel Gato, Departamento de Anatomía Humana, Facultad de Medicina, C/Ramón y Cajal 7, 47005 Valladolid, Spain. E-mail: gato@med.uva.es
}

the lens itself, the otic and nasal placodes and the neural tube, among others (Wrenn and Wessells, 1969; Bancroft and Bellairs, 1977; Brady and Hilfer, 1982; Ferreira and Hilfer, 1993); (2) a high rate of cell replication in the lens placode, with respect to the surrounding surface ectoderm, has been proposed as the mechanism responsible for creating forces involved in lens placode invagination (Modak, Morris and Yamada, 1968; Harding et al., 1971; Hendrix and Zwaan, 1974; Álvarez and Navascues, 1990; Hendrix, Madras and Johnson, 1993); (3) certain extracellular factors and, more specifically, extracellular matrix molecules, appear to be associated with epithelial invagination. What is more, it is known that alteration of the synthesis or enzyme degrading of particular matrix molecules such as collagen, certain proteoglycans and glycoproteins, may disrupt epithelial folding, as occurs in the branching of the salivary gland, lung and kidney primordia (Spooner and Faubion, 1980; Thompson and Spooner, 1983; Spooner, Bassett and Stokes, 1985; Nakanishi et al., 1986; Klein et al., 1989). In this way, it was demonstrated that proteoglycans in the extracellular matrix and basal laminas play an important role in the invagination of primordia 
similar to the lens anlage, for instance, the otic placode (Gerchman, Hilfer and Brown, 1995; MoroBalbás et al., 2000) and the neural plate (Schoenwolf and Fisher, 1983; Morris-Kay and Tuckett, 1989). In relation to this matter, there are previous studies which reveal the presence of glycoconjugates and proteoglycans in lens vesicle development. The presence of glycoconjugates on the apical surface of the lens placode during invagination and fusion was demonstrated with histochemical and lectin techniques by Van Rybroek and Olson (1981), Webster and Uknis (1987) and Yao, Alcala and Maisel (1996). More specific studies relating to proteoglycan expression during early lens development were carried out by Haloui et al. (1988). These showed that HSPG is present in the basal membrane during lens placode and vesicle stages in mice embryos. More recently, Ring, Lemmon and Halfter (1995) and Peterson et al. (1995) described the expression pattern of CSPG and hyaluronic acid during chick and macaca mulata lens development, respectively. According to the latter authors, there is a significant amount of hyaluronic acid in the matrix interposed between the optic cup and the lens placode, as well as in the intercellular spaces of lens placode cells and in the basal lamina during invagination. These authors also demonstrated the pattern of the CSPG expression in the same animal model and reported the presence of this proteoglycan in coincidence with hyaluronic acid in the matrix interposed between the optic vesicle and the lens placode; however, the presence of CSPG in the extracellular space of the lens placode seems to be restricted to the deep part of the lens epithelium, disappearing when the lens vesicle is formed. As a consequence, these authors suggest that hyaluronic acid and CSPG may be involved in the invagination and fusion of the lens placode.

In addition, there is strong evidence that FGF-2 plays a key role in lens development (McAvoy et al., 1991; Tripathi et al., 1991). It was demonstrated that, in embryonic development, there is a direct functional relationship between FGF-2 and chondroitin sulphate proteoglycan (Ruoslahti and Yamaguchi, 1991; Milev et al., 1998; Colin et al., 1999) or heparan sulphate proteoglycan (Massague, 1991; Yayon et al., 1991; Nugent and Edelman, 1992); these data suggest that the influence of proteoglycans in lens development could be mediated by growth factors.

It was mentioned above that proteoglycans may play an important role in early lens morphogenesis and, as a result, a precise knowledge of the expression pattern of proteoglycans might help to understand the mechanisms involved in lens development. In this paper the authors try, by means of immunohistochemistry and laser confocal microscopy, to throw light on the expression pattern of CSPG during lens vesicle formation in chick embryos. They also try to determine the biological role of this proteoglycan during lens vesicle formation by alteration of its synthesis with $\beta$-D-xyloside as well as its enzyme degradation with specific glycosidases.

Sulphated proteoglycans biosynthesis may be disrupted by treatment with $\beta$-D-xyloside, and this strategy has been shown to be useful as regards clarifying the biological role of proteoglycans in embryonic tissues (Yost, 1990; Alonso et al., 1999). $\beta$-D-xylosides act as artificial acceptors of xylosiltransferase, an enzyme which is involved in initiating synthesis of the glycosaminoglycans (GAGs) of certain sulphated proteoglycans (Gibson and Segen, 1977). It is known that $\beta$-D-xyloside acts upon proteoglycans in which GAGs synthesis is initiated by the incorporation of a xylose to a serine residuum in the core protein; in other words, upon CSPG and its epimere dermatan sulphate proteoglycan as well as upon heparan sulphate proteoglycan (HSPG) and heparin (Sobue et al., 1987; Hahn and Birk, 1992). Furthermore, it has been shown that the activity of $\beta$-D-xyloside is dosedependent, acting preferentially in small doses upon CSPG biosynthesis and in large doses upon HSPG biosynthesis (Lugenwa and Esko, 1991). In order to differentiate the biological role of these proteoglycans during lens vesicle formation chondroitinase AC is used, which specifically digests CSPG (but not dermatan sulphate), and type II heparinase, a specific enzyme for HSPG/heparin.

\section{Materials and Methods}

Fertile white leghorn eggs were incubated at $38^{\circ} \mathrm{C}$ in a humidified atmosphere to obtain chick embryos at different developmental stages ranging from 14 to 17 H.H. (Hamburger and Hamilton, 1951), involving the transition from lens placode to lens vesicle. Embryos were removed from the extraembryonic membranes and fixed for $6 \mathrm{hr}$ in Bouin's fluid at room temperature. After dehydration in graded ethanol series and embedding in paraplast, $8 \mu \mathrm{m}$ transverse sections were stained with haematoxylin and eosin. The most representative sections were photographed with a Nikon microphot-FXA photomacroscope.

\section{Immunohistochemistry}

Chick embryos of developmental stages similar to those used for histological study were immersed for $1 \mathrm{hr}$ in Carnoy's fixative at room temperature and gently soaked, after which they were dehydrated in graded ethanol series and embedded in paraplast. Carnoy's fixative has been reported to be the best for maintaining the carbohydrate epitope of proteoglycans (Gato et al., 1993).

Deparaffinized sections of $8 \mu \mathrm{m}$ from five different lens primordia at each stage were washed in phosphate-buffered saline (PBS), pre-incubated with normal horse serum (1/20 in PBS) and incubated overnight with anti-chondroitin sulphate monoclonal antibody CS-56 (Sigma). After two washings in PBS, 
the sections were reincubated for $30 \mathrm{~min}$ in fluorescein-conjugated goat antimouse IgM (Vector) as a secondary antibody, mounted in Aquamount (Gurr) and studied under a Zeiss LSCM 310 laser confocal microscope. Control sections were prepared as described above but pre-immune serum was used as the primary antibody and no labelling was observed.

In certain embryos HSPG expression was determined by a procedure similar to the one mentioned above: the primary antibody was a rat Ig-G antiheparan sulphate proteoglycan (Upstate Biotechnology) and the secondary antibody was an anti-rat Ig-G FITC conjugate (Sigma).

\section{$\beta$-D-xyloside Treatment}

Treatment was carried out 'in ovo' after 40-45 hr of incubation. A small window was opened in the shell and the embryos were classified according to Hamburger and Hamilton (1951) parameters. For $\beta$-D-xyloside treatment, a single dose of $24 \mu \mathrm{l} p$-nitophenyl- $\beta$-D-xylopyranoside $4 \mathrm{~mm}$ (Sigma) was injected subgerminally, with a Hamilton microsyringe, into 11 H.H. stage embryos prior to lens placode formation. These doses were chosen as a result of previous studies that showed a clear disruption in CSPG biosynthesis without severe effects on chick embryo development and protein synthesis (Moro-Balbás et al., 1998; Alonso et al., 1999). Control embryos were injected with sterile saline solution or with $\alpha$-D-xyloside $4 \mathrm{~mm}$ (an inactive anomer of $\beta$-D-xyloside). The aim of this group was to rule out possible direct effects of xylosides on embryo development and growth. Afterwards, the opening in the shell was sealed and the eggs were reincubated for $12 \mathrm{hr}$ until the embryos reached stage 17 H.H. when the lens vesicle was isolated from the surface ectoderm. Under these conditions, a series of 20 treated and 20 control embryos was employed in our study. These embryos were fixed and processed as described above for histological and immunohistochemical study.

\section{Enzymes Microinjection}

Microinjecting was carried out 'in ovo', subsequent to the opening of a small window in the egg shell, then the embryos were classified by age. Only the 12 H.H. stage embryos were microinjected. Once the vitelline membrane had been cut with tungsten needles, subectodermal microinjection was undertaken, with $2 \mathrm{nl}$ of a solution of $10 \mathrm{U}$ heparinase II from Flavobacterium heparinum (Sigma) dissolved in $25 \mu \mathrm{l}$ of PBS, or $2 \mathrm{nl}$ of a solution of $8 \cdot 7 \mathrm{U}$ chondroitinase $\mathrm{AC}$ (Sigma) dissolved in $500 \mu \mathrm{l}$ of PBS, on the right side of the eye anlage. On the left side, used as the control, the same amount of heat-deactivated enzymes were microinjected. Microinjection was effected with $10 \mu \mathrm{m}$ tip diameter microneedles connected to a microinjector (Medical Systems Corp, Greenvale, NY
11548, U.S.A., PLI-100). When microinjection had been completed, the egg shell was sealed with a plastic adhesive tape and we proceeded to re-incubate for a period of $24 \mathrm{hr}$, until the embryos reached stage 16-17 H.H. A total of 20 embryos were microinjected with each enzyme. Upon extraction, the microinjected embryos were processed for HSPG and CSPG immunomarking, following the same method as the one described above. The effect produced by treatment with enzymes was checked by comparison with the treated side (right) relative to the control side (left).

\section{Results}

Evolutive Pattern of CSPG Immunolabelling during Lens Vesicle Development

In the very early stages of development, prior to the appearance of the lens placode, no differences were found in the expression pattern of CSPG between the presumptive lens ectoderm and the rest of the surface ectoderm (not shown). In coincidence with the formation of the lens placode and its invagination, fusion and isolation from the surface ectoderm, significant changes appear in the distribution of CSPG immunolabelling with respect to the surrounding ectoderm. These changes suggest that this proteoglycan could be involved in early lens morphogenesis.

In this study the authors were able to verify CSPG expression at four different locations of the lens primordium, each one developing independently of the others. These were: (1) immunolabelling on the apical surface; (2) immunolabelling on the basal lamina; (3) intercellular immunolabelling in the lens epithelium; and (4) immunolabelling in the fusion region of the lens placode edges.

Apical surface CSPG immunolabelling. At this level, immunolabelling appeared as a fine lamina of immunoreactive material covering the apical surface of the lens epithelium cells, which suggests that it may form part of a 'cell coat'. The presence of this positive CSPG material began with the appearance of the lens placode [Fig. 1(A)] and represented the first sign of differentiation in CSPG expression with respect to the surrounding surface ectoderm. At this stage, CSPG had the features of a fine discontinuous lamina of moderately immunoreactive material. This apical immunolabelling varied in terms of its presence and intensity particularly at stages prior to lens vesicle formation; this suggests that it might be lost due to diffusion during embryo processing [compare Fig. $1(\mathrm{~A}-\mathrm{C})$ ]. Once the lens vesicle had formed, apical CSPG immunolabelling was restricted to the posterior epithelium and appeared as a fine continuous lamina of greater intensity than at previous stages [Figs $1(\mathrm{~F})$ and 2(A)]. 

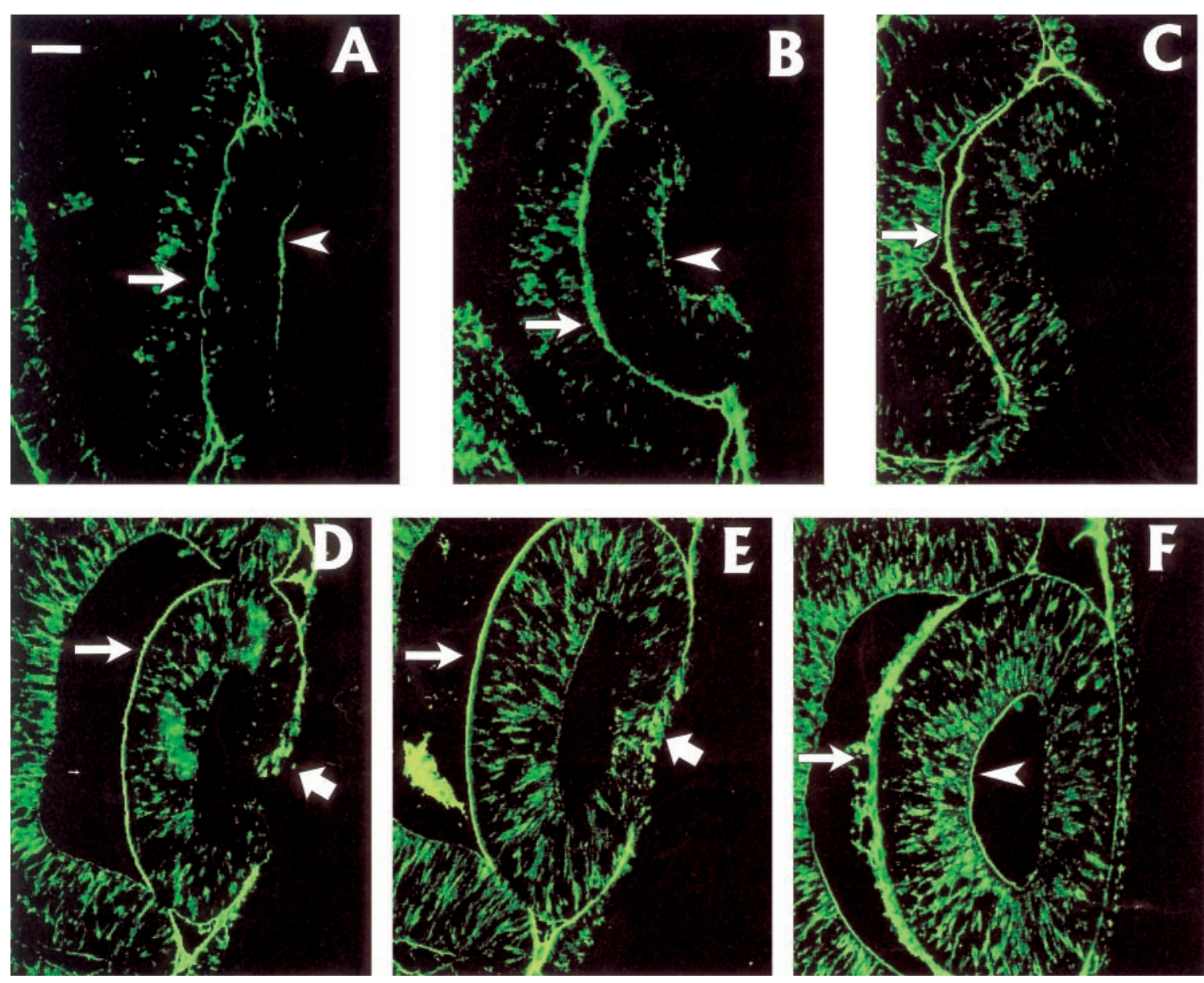

FIg. 1. Micrographs at lens primordium level of chick embryos processed with CS56 anti-chondroitin sulphate antibody and using a conjugate secondary antibody with fluorescein isothiocyanate (FITC). The images show the CSPG expression pattern during formation of the lens vesicle. (A) Lens placode phase, Stage 14 H.H. (B and C) Invagination phase, Stage 15 H.H. (D and E) Approximation-fusion phase and lens stalk formation Stage 16 H.H. (F) Lens vesicle independent of surface ectoderm, Stage 17 H.H. Note CSPG immunolabelling on the apical surface of the lens primordium (arrow heads) and on the basal surface (small arrows), a progressive increase in intercellular CSPG immunolabelling on the lens epithelium and more intense immunomarking in the fusion and lens stalk regions (large arrows). All images have the same magnification. Bar $=20 \mu \mathrm{m}$.

Immunolabelling on the basal lamina. No significant differences in the lens basal lamina immunolabelling were encountered with respect to the rest of the surface ectoderm. This immunolabelling had the form of a continuous and well-defined lamina of greater intensity than apical CSPG immunomarking, extending from the placode phase to that of the lens vesicle (Fig. 1). At the lens placode stage, CSPG immunolabelling on the basal lamina was accompanied by delineation of the optic vesicle-cup in the regions in which the two structures came into contact [Fig. 1(A) and (B)]. However, in the lens vesicle stage, in the area of contact between the edges of the optic cup and the lens vesicle equator, CSPG immunolabelling was significantly less or even disappeared [Fig. 1(D) and (E)]. Highly immunoreactive material in the primitive vitreous chamber was frequently observed, and this was occasionally seen adhering to the CSPG on the lens basal lamina [Figs $1(\mathrm{~F})$ and $2(\mathrm{~A})]$.

Intercellular immunolabelling in lens epithelium. In this study detected a progressive increase in intercellular
CSPG immunolabelling in the lens primordium epithelium was detected. Whilst during the lens placode stage [Fig. 1(A)], there was barely any CSPG expression inside the placodial epithelium, at the start of invagination there was a significant increase in intercellular immunolabelling [Fig. 1(B) and (C)] which continued into later stages. Once lens vesicle formation had taken place, CSPG immunolabelling became considerably more intense at the posterior epithelium level of the lens vesicle [Fig. 1(D-F)]. Intercellular CSPG expression was seen to behave similarly in the optic vesicle epithelium. Once again, a notable increase in immunolabelling took place, coinciding with the start of the optic vesicle invagination process leading to the formation of the optic cup (Fig. 1).

Immunolabelling in the fusion region of the lens placode edges. Coinciding with the approximation and fusion of the lens placode edges, observation was made of a local increase in intercellular CSPG immunolabelling in the fusion and lens stalk regions, not seen in the rest of the future lens vesicle's anterior epithelium [Fig. 1(D) and 

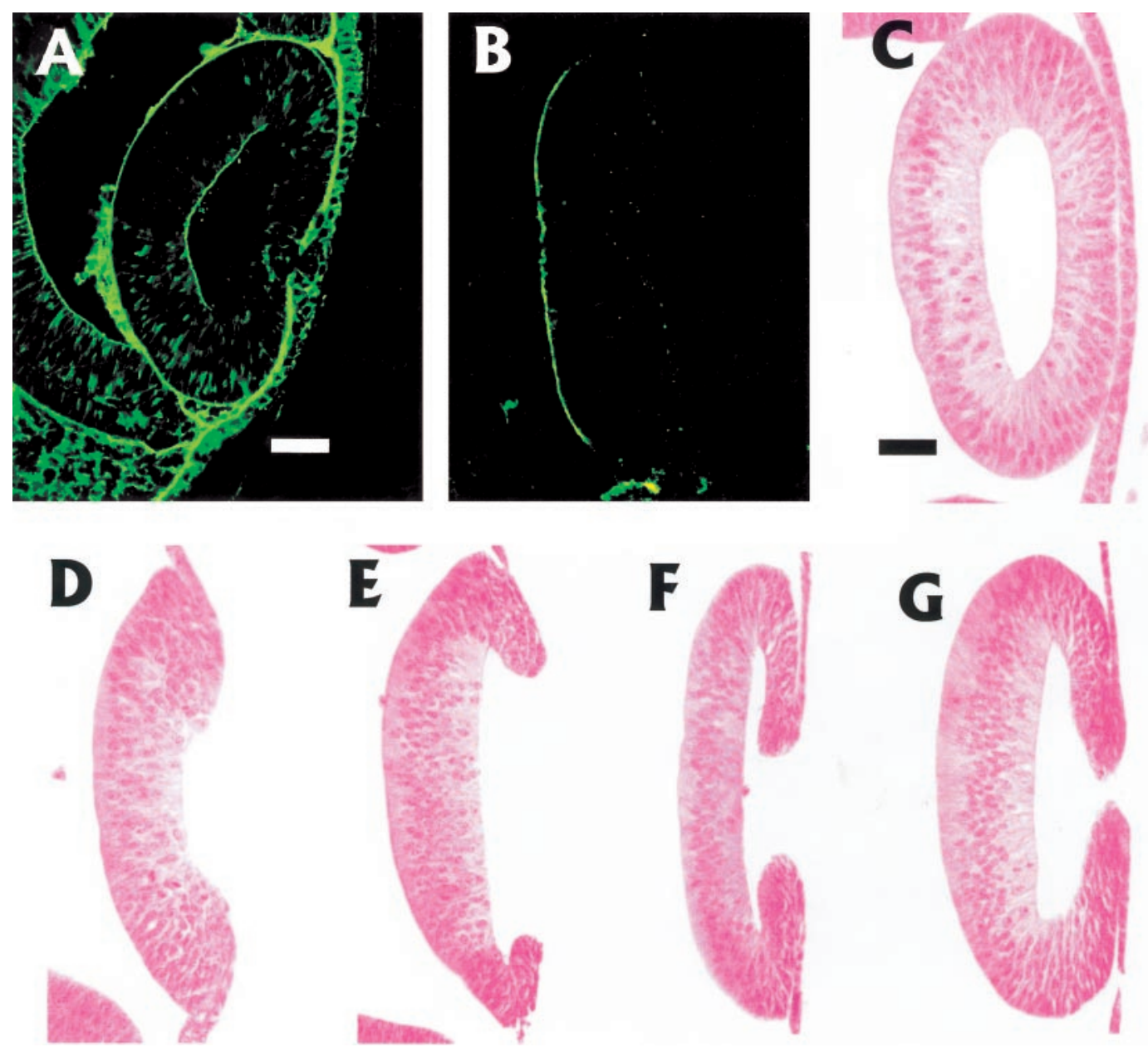

FIG. 2. Comparison of the lens primordium control embryos of 16-17 H.H. stages and embryos at the same stages treated with $\beta$-D-xyloside. Note the sharp decrease in CSPG immunolabelling among embryos treated with $\beta$-D-xyloside (B) with respect to control (A). The haematoxylin-eosin stained histological sections of 17 H.H.-stage chick embryo lens primordium show alterations induced by treatment with $\beta$-D-xyloside $(D-G)]$, compared with control embryos $(C)$ in which the lens primordium has reached the lens vesicle phase. In embryos treated with $\beta$-D-xyloside it is possible to observe different degrees of alteration, ranging from total absence of lens placode invagination (D), partial invagination ( $\mathrm{E}$ and $\mathrm{F}$ ), or disruptions in fusion of the edges (G). Bar in (A) and (B) $=23 \mu \mathrm{m}$. Bar in (C), (D), (E), (F) and (G) $=25 \mu \mathrm{m}$.

(E)]. This increased CSPG expression was a transitory process, since it disappeared once the edges of the lens placode had joined together and immunolabelling intensity became uniform in the whole of the anterior lens epithelium [Fig. 1(F)].

\section{Effect of CSPG Synthesis Disruption or Enzymatic Digestion on the Invagination and Fusion of the Lens Placode}

In order to ascertain the role of the CSPG during lens vesicle formation, initially the synthesis of this proteoglycan was disrupted by means of 'in ovo' subgerminal administration of $\beta$-D-xyloside, a compound which disrupts the synthesis of sulphated proteoglycans, not only preferentially CSPG but also HSPG (Sobue et al., 1987; Lugenwa and Esko, 1991). The control group was injected with a sterile Ringer solution or with a solution of $\alpha$-D-xyloside (an inactive anomer of $\beta$-D-xyloside). Secondly, to ensure that the effect of $\beta$-D-xyloside is exclusively the result of interference in CSPG synthesis, the authors conducted 'in vivo' enzymes digestion with specific glycosidases, chondroitinase AC for CSPG and heparinase II for HSPG.

$\beta$-D-xyloside treatment. CSPG immunolabelling showed the effectiveness of $\beta$-D-xyloside in CSPG synthesis disruption in the lens anlage. The group of control embryos showed a normal degree of lens development and a CSPG expression pattern similar to that already mentioned in connection with the lens vesicle [Fig. 2(A)]. However, embryos treated with $\beta$ D-xyloside [Fig. 2(B)] showed, apart from morphological alterations in invagination and fusion, notable CSPG depletion throughout the entire lens primordium, and only slight CSPG immunolabelling was apparent at the basal lamina level of the posterior epithelium. These findings demonstrate that treatment with $\beta$-D-xyloside considerably reduces the CSPG synthesis capacity of the lens primordium although also detected was a reduction in the expression of 
HSPG present in the basal lamina of the lens primordium (not shown).

The results show that treatment with $\beta$-D-xyloside, administered prior to the appearance of the lens placode, does not alter its formation, suggesting that CSPG/HSPG plays no important role in this process. Nevertheless, whilst lens primordium development was normal amongst control embryos [Fig. 2(C)], it was observed that in all the embryos treated with $\beta$-Dxyloside notable alterations took place in lens primordium invagination and fusion [Fig. 2(D), (E) and (G)]; these alterations were bilateral in most cases. Three different disruption patterns were encountered in lens primordium morphogenesis, induced by $\beta$-D-xyloside. In the most affected embryos the lens primordium was at the lens placode stage, barely showing signs of having commenced the invagination process [Fig. 2(D)]; yet the lens epithelium appeared, as a result of its size, to have continued growing. Among a second group of embryos treated with $\beta$-D-xyloside, the invagination process had started but its development was abnormal, with only slight growth of the anterior epithelium and a wide anterior opening of the lens vesicle, suggesting that there had been a break in the mutual approximation of the edges of the primordium [Fig. 2(E) and (F)]; in addition, a flattering in the curvature of the posterior epithelium with respect to the control embryos was observed [Fig. 2(C)], characteristic of this stage of development. In the last group of embryos treated with $\beta$-D-xyloside disruptions were less evident; the lens placode edges did not come into contact and a small lens pore persisted [Fig. 2(G)], or contact was not effective enough to bring about the fusion of the edges. As in the previous group, anterior epithelium growth was seen to be less than among control embryos.

Enzymatic digestion. With a view to ascertaining whether the alterations induced by $\beta$-D-xyloside were the result of interference in CSPG synthesis, enzymatic digestion was conducted with chondroitinase AC and type II heparinase of the two proteoglycans (CSPG and HSPG) present during lens vesicle formation and possibly affected by the $\beta$-D-xyloside.

In the embryos microinjected with chondroitinase $\mathrm{AC}$, the lens primordium (right) showed alterations in the invagination and fusion processes similar to those described when treatment with $\beta$-D-xyloside was performed. Among the most affected embryos, the lens primordium remained at the placode stage [Fig. 3(A)], whilst in other cases invagination continued but did not come to completion [Fig. 3(B)]. On the control side (left), invagination took place normally (not shown). The effectiveness and specificity of the enzyme treatment was tested by CSPG immunolabelling. In the lens primordia microinjected with chondroitinase AC, expression of CSPG was severely diminished [Fig. 3(C)], whereas there was no alteration in HSPG expression (not shown).
Microinjection with type II heparinase brought about no evident alterations in invagination and fusion [Fig. 3(D)], and the morphology of the treated lens primordium (right) was similar to that of the control (left). Immunolabelling with anti-HSPG antibody revealed a clear reduction in HSPG on the treated side [Fig. 3(F)] with respect to the control side which showed an intense immunolabelling on the basal lamina of lens vesicle [Fig. 3(E)]. In the embryos microinjected with type II heparinase, no alteration in CSPG expression was perceived (not shown), which demonstrates the specificity of this enzyme on HSPG digestion.

\section{Discussion}

Our findings demonstrate that there are specific variations in CSPG expression from the appearance of the lens primordium up to the formation of the lens vesicle. We have shown that during lens vesicle formation CSPG is present in four different locations: (1) a cell coat covering the apical surface of the lens primordium cells; (2) on the basal lamina of the lens placode and vesicle; (3) in the intercellular spaces of the lens epithelium; and (4) in the fusion and lens stalk regions. The expression pattern of CSPG and especially its intercellular and apical positioning, suggest that the lens epithelial cells are the ones responsible for its secretion. In this regard it has been demonstrated that the embryonic ectoderm and certain epithelia derived from it have the capacity to synthesize and secrete this proteoglycan (Fichard et al., 1991; Gato et al, 1993; Alonso et al., 1998a). In addition, interference in CSPG expression produces absence or abnormal invagination and defects in the fusion of the lens placode edges, suggesting that CSPG plays an important role in early lens morphogenesis. Although $\beta$-D-xyloside may disrupt CSPG and HSPG synthesis, alterations induced by $\beta$-D-xyloside were reproduced only by chondroitinase $\mathrm{AC}$ and not by type II heparinase; this would enable us to attribute the effects of the $\beta$-D-xyloside to interference in the CSPG synthesis.

The presence of CSPG covering the apical surface of the lens placode cells suggests the existence of a 'cell coat' which continues up to the lens vesicle stage particularly in the posterior epithelium. It has been postulated (Van Rybroek and Olson, 1981; Yao et al., 1996.) that an apical 'cell coat' rich in glycoconjugates might play a significant role in lens epithelium invagination. Moreover, it was shown that, in Aphakic strain mice with alterations in the composition of the apical 'cell coat' glycosaminoglycans there are serious defects in early lens development (Zwaan and Webster, 1984). It is not known how these covering glycoconjugates are able to intervene in epithelial invagination, although it has been suggested that these molecules may bind and concentrate large amounts of $\mathrm{Ca}^{++}$near the apical surface of the 

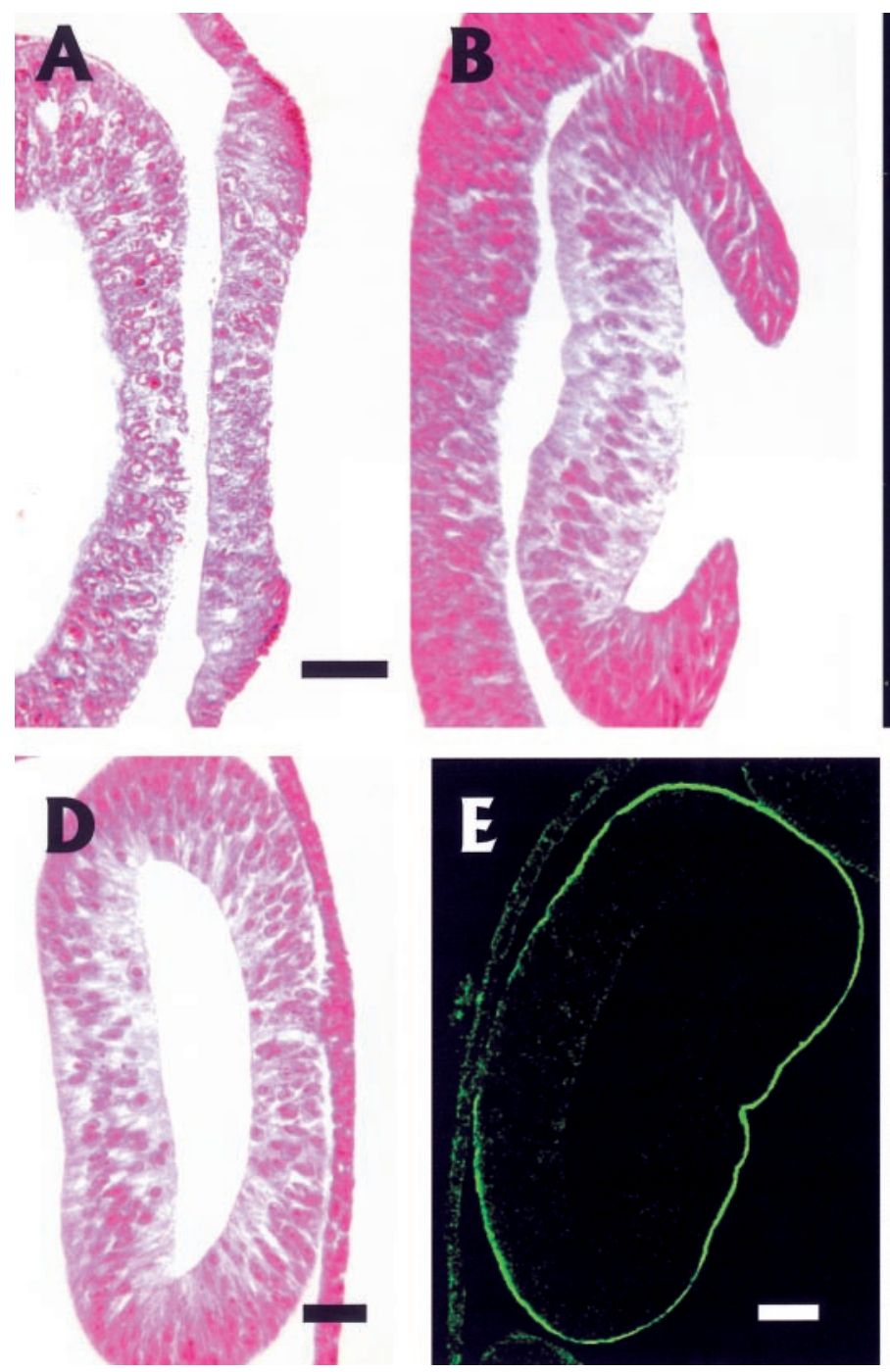
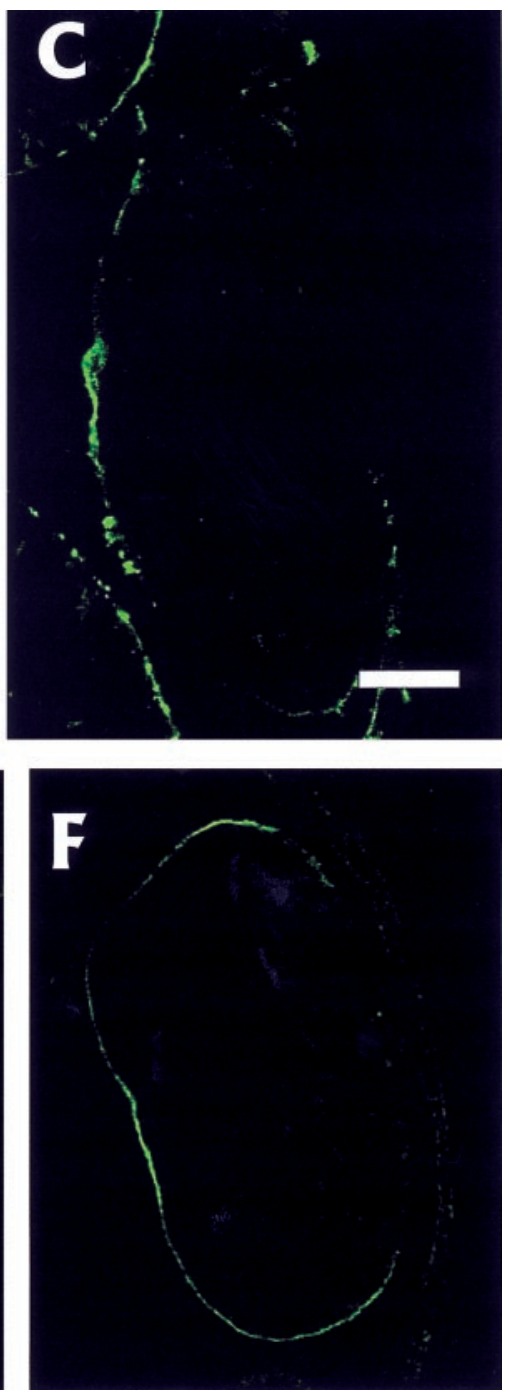

FIG. 3. Effect of specific glycosidase digestion on lens vesicle formation. Haematoxilin-eosin stained histological sections [(A) and (B)] of 16 H.H.-stage chick embryos lens primordium show alterations induced by digestion with chondroitinase AC. CSPG immunolabelling of a similar section shows the effectiveness of this enzymatic treatment (C); compare this image with [Fig. 1(E) and $(\mathrm{F})$ ]. Lens vesicle formation is not disrupted by type II heparinase treatment (D). The normal pattern of HSPG immunolabelling in a control lens vesicle (left eye anlage) is shown in (E) and the effectiveness of type II heparinase treatment can be observed in (F) (right eye anlage). Bar in (A) and (B) are common $(25 \mu \mathrm{m})$. Bars in (C) and (D) $=25 \mu \mathrm{m}$. Bar in (E) and (F) are common $(25 \mu \mathrm{m})$.

above-mentioned cells and activate contraction of the apical microfilaments involved in cell form changes (Yang and Hilfer, 1982; Slepecky and Chamberlain, 1985; Turley, Brassel and Moore, 1990).

In addition, the presence of intense CSPG immunomarking on the basal lamina of the lens primordium during the entire study period has been demonstrated. This could be related to its invagination since the drastic reduction in CSPG on the basal lamina induced by $\beta$-D-xyloside or chondroitinase AC, but not by type II heparinase, was accompanied by serious defects in lens primordium invagination. In this regard, it has been shown that proteoglycans present in the basal lamina, play an important role in the invagination of numerous embryonal epithelial primordia (Klein et al., 1989; Gerchman et al., 1995; Toriyama et al., 1997; Moro-Balbás et al., 2000). It has been suggested that the basal lamina might favour the establishing of connections between the cellular cytoskeleton and the extracellular matrix (Goetinck 1991; Jalkanen, Jalkanen and Bernfield, 1991), which would lead to changes in cell form, thereby conditioning epithelial invagination. In later stages of development, when the lens fibres can begin to be differentiated, it has been shown that basal lamina proteoglycans assume an important role in regulating the processes of cellular replication, cell migration and the subsequent differentiation of the lens fibres; this is due to the fact that the lens capsule proteoglycans intervene in the storing and activation of fibroblast growth factor 2, modulating its bioavailability (Zwaan and Webster, 1984; Haloui et al., 1988; Chamberlain and McAvoy, 1997).

In addition, it was observed that basal lamina CSPG in the lens vesicle diminished and even disappeared in the regions where contact was made with the edges of 
the optic cup. The influence exerted by these edges on differentiation of the lens fibres has been reported (Tripathi et al., 1991; Wride, 1996), and it is possible that the disappearance or diminishing of CSPG in this region favours contact between both structures and facilitates some influence of the optic cup edges on the lens primordium at this stage of development.

In this study the authors have shown that intercellular CSPG increases progressively during the invagination process of the lens epithelium and their experimental reduction brings about serious disruptions in this process. CSPG intercellular localization was previously described by Peterson et al. (1995) in macaca mulata embryos; however, in this species intercellular CSPG disappears in the lens vesicle phase, unlike what occurs in chick embryos. The large hydrophilic capacity of proteoglycans in biological solutions has already been reported (Comper and Laurent, 1978; Comper and Zamparo, 1990) and it has been suggested that they produce a volume increase in the intercellular spaces which favours epithelial flexibility and consequently facilitates invagination as has been demonstrated in neural plate (Schoenwolf and Fisher, 1983; Morris-Kay and Tuckett, 1989; Alonso et al., 1998a) and otic primordium (Gerchman et al., 1995) invagination. In these primordia maximum folding regions have been described in which abrupt changes in epithelial direction take place (Meier, 1978; Hilfer, Esteves and Sanzo, 1989; Schoenwolf and Smith, 1990). In this sense, on the lens placode, regions of maximum folding appear in the equatorial area which coincides with an increase in intercellular CSPG expression. Moreover, experimental alteration in CSPG expression induced by us produced in many cases significant defects in lens placode invagination.

In some $\beta$-D-xyloside and chondroitinase AC treated embryos, lens primordium invagination did take place but, as mentioned above, there appeared to be defective fusion of the edges. At this level there was a transitory increase in CSPG immunolabelling intensity, coinciding with the fusion process and lens stalk formation. This suggests that CSPG might play a role in the recognition and adherence of the edges of the lens placode. In this regard it was demonstrated that glycoproteins are present in the intercellular matrix interposed between the edges of the lens placode during its apposition (Van Rybroek and Olson, 1981; Yao et al., 1996). Similarly, the presence of a matrix rich in glycoconjugates interposed in the epithelial fusion region was also reported during the fusion of other embryonal primordia such as the neurla tube (Smits-van-Prooije et al., 1986; Trasler and Morriss-Kay, 1991) and the palate (Martínez-Alvarez et al., 2000). What is more, in the case of the neural primordium, experimental evidence showed that disruption in the synthesis or enzyme degradation of the proteoglycans seriously alters the fusion process (Morris-Kay and Crutch,
1982; Morris-Kay and Tuckett, 1989; Alonso et al. 1998b).

To conclude, this paper offers experimental evidence that the CSPG present during the early stages of lens morphogenesis is involved in epithelial invagination and fusion leading to lens vesicle formation in chick embryos. The importance of sulphated proteoglycans in the very early stages of this primordium's development indicates that further studies should be carried out in order to clarify the role of these molecules at other stages of lens morphogenesis in the immediately subsequent phases.

\section{Acknowledgements}

The authors thank Dr David Rixham for language translation assistance, and Sagrario Callejo and Pilar Martín for technical support. This work was supported by a grant from the Ministerio de Educación y Cultura, Programa sectorial de promoción general del conocimiento PB98-1635, and by a grant from Junta de Castilla y León Va35/00b.

\section{References}

Alonso, M. I., Gato, A., Moro, J. A. and Barbosa, E. (1998a). Disruption of proteoglycans in neural tube fluid by $\beta$-Dxyloside alters brain enlargement in chick embryos. Anat. Rec. 252, 499-508.

Alonso, M. I., Gato, A., Moro, J. A., Martín, P. and Barbosa, E. (1998b). Neural tube defects induced by $\beta$-D-xyloside: evidence of a role for sulfated proteoglycans in neural fold fusion in rat embryos. Eur. J. Anat. 2, 133-40.

Alonso, M. I., Gato, A., Moro, J. A., Martín, P. and Barbosa, E. (1999). Involvement of sulfated proteoglycans in embryonic brain expansion at earliest stages of development in rat embryos. Cells Tiss. Org. 165, 1-9.

Álvarez, I. S. and Navascues, J. (1990). Shaping, invagination, and closure of the chick embryo otic vesicle: scanning electron microscopic and quantitative study. Anat. Rec. 228, 315-26.

Bancroft, M. and Bellairs, R. (1977). Placodes of the chick embryo studies by SEM. Anat. Embryol. Berl. 151, 97-108.

Brady, R. C. and Hilfer, S. R. (1982). Optic cup formation: a calcium regulated process. Proc. Natl Acad. Sci., U.S.A. 79, 5587-91.

Chamberlain, C. G. and McAvoy, J. W. (1997). Fibre differentiation and polarity in the mammalian lens: a key role for FGF. Prog. Retin. Eye. Res. 16, 443-78.

Colin, S., Jeanny, J. C., Mascarelli, F., Vienet, R., AlMahmood, S., Curtois, Y. and Labarre, J. (1999). In vivo involvement of heparan sulfate proteoglycan in the bioavailability, internalization and catabolism of exogenous basis fibroblast growth factor. Mol. Pharmacol. 55, 74-82.

Comper, W. D. and Laurent, T. C. (1978). Physiological function of connective tissue polysaccharides. Physiol. Rev. 58, 255-315.

Comper, W. D. and Zamparo, O. (1990). Hydrodynamic properties of connective-tissue polysaccharides. Biochem. J. 269, 561-4.

Ferreira, M. C. and Hilfer, S. R. (1993). Calcium regulation of neural fold formation; visualization of the actin cytoskeleton in living chick embryos. Dev. Biol. 427, 427-40.

Fichard, A., Verna, J. M., Olivares, J. and Saxod, R. (1991). Involvement of a chondroitin sulfate proteoglycan in 
the avoidance of chick epidermis by dorsal root ganglia fibers: a study using beta-D-xyloside. Dev. Biol. 148, $1-8$.

Gato, A., Moro, J. A., Alonso, M. I., Pastor, J. F., Represa, J. J. and Barbosa, E. (1993). Chondroitin sulphate proteoglycan and embrionic brain enlargement in the chick. Anat. Embryol. 188, 101-6.

Gerchman, E., Hilfer, S. R. and Brown, J. W. (1995). Involvement of extracellular matrix in the formation of the inner ear. Dev. Dyn. 202, 421-32.

Gibson, K. D. and Segen, B. J. (1977). The mode of action of 4-methyllumbelliferil $\beta$-D-xyloside and the synthesis of condroitin sulfate in embryonic chicken sternum. Biochem. J. 168, 65-79.

Goetinck, P. F. (1991). Proteoglycans in development. Curr. Top. Dev. Biol. 25, 111-31.

Hahn, R. and Birk, D. (1992). $\beta$-D-xyloside alters dermatan sulfate proteoglycan synthesis and the organization of developing avian corneal stroma. Development 115 , 383-93.

Haloui, Z., Jeanny, J. C., Jonet, L., Curtois, Y. and Laurent, M. (1988). Immunochemical analysis of extracellular matrix during embryonic lens development of the cat fraser mouse. Exp. Eye Res. 46, 463-74.

Hamburger, V. and Hamilton, H. L. (1951). A series of normal stages in the development of the chick embryo. J. Morphol. 88, 49-92.

Harding, C. V., Reddan, J. R., Unakar, N. J. and Bagchi, M. (1971). The control of cell division in the ocular lens. Int. Rev. Cytol. 31, 215-300.

Hendrix, R., Madras, N. and Johnson, R. (1993). Growth pressure can drive early chick lens geometries. Dev. Dyn. 196, 153-64

Hendrix, R. W. and Zwaan, J. (1974). Cell shape regulation and cell cycle in embryonic lens cells. Nature 247, $145-7$.

Hilfer, S. R. (1983). Development of the eye of chick embryo. Scan. Electron. Microsc. 3, 1353-69.

Hilfer, S. R., Esteves, R. A. and Sanzo, J. F. (1989). Invagination of the otic placode: normal development and experimental manipulation. J. Exp. Zool. 251, 253-64.

Jalkanen, M., Jalkanen, S. and Bernfield, M. (1991). Binding of extracellular effector molecules by cell surface proteoglycans. In Receptors for Extracellular Matrix. (Mcdonald, J. A. and Mecham, R. P., Eds). Pp. 1-37. Academic Press: San Diego, CA, U.S.A.

Klein, D. J., Brown, D. M., Moran, A., Oegema, T. R., Jr. and Platt, J. L. (1989). Chondroitin sulfate proteoglycan synthesis and reutilization of $\beta$-D-xyloside-initiated chondroitin/dermatan sulfate glycosaminoglycans in fetal kidney branching morphogenesis. Dev. Biol. 133. 515-28.

Lugenwa, F. N. and Esko, J. D. (1991). Estradiol $\beta$-D-xyloside, an efficient primer for heparan sulfate biosynthesis. J. Biol. Chem. 266, 6674-7.

Martínez-Alvarez, C., Bonelli, R., Tudela, C., Gato, A., Mena, J., O'Kane, S. and Ferguson, M. W. (2000). Building medial edge epithelial cells and palatal fusion. Int. J. Dev. Biol. 44, 331-5.

Massague, J. (1991). A helping hand from proteoglycans. Cell. Growth Factors 1, 117-9.

McAvoy, J. W., Chamberlain, C. G., de Iongh, R. U., Richardson, N. A. and Lovicu, F. J. (1991). The role of fibroblast growth factor in eye lens development. Ann. N.Y. Acad. Sci. 638, 265-74.

Meier, S. (1978). Development of the embryonic chick otic placode. I. Light microscopic analysis. Anat. Rec. 191, $447-58$.
Milev, P., Monnerie, H., Popp, S., Margolis, R. K. and Margolis, R. U. (1998). The core protein of the chondroitin sulfate proteoglycan phosphocan is a high-affinity ligand of fibroblast growth factor- 2 and potentiates its mitogenic activity. J. Biol. Chem. 273. 21439-42.

Modak, S. P., Morris, G. and Yamada, T. (1968). DNA synthesis and mitotic activity during early development of chick lens. Dev. Biol. 17, 544-61.

Moro-Balbás, J. A., Gato, A., Alonso, M. I. and Barbosa, E. (1998). Local increase level of chondroitin sulfate induces changes in the rhombencephalic neural crest migration. Int. J. Dev. Biol. 42, 207-16.

Moro-Balbás, J. A., Gato, A., Alonso, M. I., Martín, P. and de la Mano, A. (2000). Basal lamina heparan sulphate proteoglycan is involved in otic placode invagination in chick embryos. Anat. Embryol. 202, 333-43.

Morris-Kay, G. M. and Crutch, B. (1982). Culture of rat embryos with $\beta$-D-xiloside: evidence of a role for proteoglycans in neurulation. J. Anat. 134, 491-506.

Morris-Kay, G. M. and Tuckett, F. (1989). Immunohistochemical localisation of chondroitin sulphate proteoglycans and the effects of chondroitinase $A B C$ in 9 to 11-day rat embryos. Development 106, 787-98.

Nakanishi, Y., Sugiura, F., Kishi, J. and Hayakawa, T. (1986). Local effects of implanted Elvax chips containing collagenase inhibitor and bacterial collagenase on branching morphogenesis of mouse embryonic submandibular glands in vitro. Zool. Sci. 3, 479-86.

Nugent, M. A. and Edelman, E. R. (1992). Kinetics of basic fibroblast growth factor binding to its receptor and heparan sulfate proteoglycan: a mechanism for cooperativity. Biochemistry 31, 8876-83.

Peterson, P. E., Pow, C. S., Wilson, D. B. and Hendrickx, A. G. (1995). Localization of glycoproteins and glycosaminoglycans during early eye development in the macaque. J. Anat. 186, 31-42.

Ring, C., Lemmon, V. and Halfter, W. (1995). Two chondroitin sulfate proteoglycans differentially expressed in the developing chick visual system. Dev. Biol. 168, 11-27.

Ruoslahti, E. and Yamaguchi, Y. (1991). Proteoglycans as modulators of growth factor activities. Cell 64, 867-9.

Schoenwolf, G. C. and Fisher, M. (1983). Analysis of the effects of streptomyces hyalurinidase on formation of the neural tube. J. Embryol. Exp. Morphol. 73, 1-15.

Schoenwolf, G. C. and Smith, J. L. (1990). Mechanisms of neurulation: traditional viewpoint and recent advance. Development 109, 243-72.

Schook, P. (1980a). Morphogenetic movements during the early development of the chick embryo. A light microscopic and spatial reconstructive study. Acta Morphol. Neerl. Scand. 18, 1-30.

Schook, P. (1980b). Morphogenetic movements during the early development of the chick eye. An ultrastructural and spatial reconstructive study. A. Invagination of the lens placode. Acta Morphol. Neerl. Scand. 18, 133-57.

Slepecky, N. and Chamberlain, S. C. (1985). Immunoelectron microscopic and immunofluorescent localization of cytoskeletal and muscle-like contractile proteins in inner ear sensory hair cells. Hear. Res. 20, 245-60.

Smits-van-Prooije, A. E., Poelman, E., Gesink, A. F., VanGroeningen, M. J. and Vermeij-Keers, C. (1986). The cell surface coat in neurulating mouse and rat embryos, studied with lectins. Anat. Embryol. Berl. 175, 111-7.

Sobue, M., Habuchi, H., Ito, K., Yonekura, H., Oguri, K., Sakurai, K., Kamohara, S., Ueno, Y., Noyori, R. and Suzuki, S. (1987). $\beta$-D-xylosides and their analogues as artificial initiators of glycosaminoglycan chain syn- 
thesis. Aglycone-related variation in their effectiveness in vitro and in ovo. Biochem. J. 241, 591-601.

Spooner, B. S. and Faubion, J. (1980). Collagen involvement in branching morphogenesis of embryonic lung and salivary gland. Dev. Biol. 77, 84-102.

Spooner, B. S., Bassett, K. and Stokes, B. (1985). Sulfated glycosaminoglycan deposition and processing at the basal epithelial surface in branching and in $\beta$-Dxyloside-inhibited embryonic salivary glands. Dev. Biol. $109,177-83$.

Thompson, H. A. and Spooner, B. S. (1983). Proteoglycan and glycosaminoglycan synthesis in embryonic mouse salivary glands: effects of $\beta$-D-xyloside, an inhibitor of branching morphogenesis. J. Cell. Biol. 96, 1443-50.

Toriyama, K., Muramatsu, H., Hoshino, T., Torii, S. and Muramatsu, T. (1997). Evaluation of heparinbinding growth factors in rescuing morphogenesis of heparitinase-treated mouse embryonic lung explans. Differentiation 61, 161-7.

Trasler, D. G. and Morriss-Kay, G. (1991). Immunohistochemical localization of chondroitin and heparan sulfate proteoglycans in pre-spina bifida splotch mouse embryos. Teratology 44, 571-9.

Tripathi, B. J., Tripathi, R. C., Livingston, A. M. and Borisuth, N. S. C. (1991). The role of growth factors in the embryogenesis and differentiation of the eye. Am. J. Anat. 192, 442-71.

Turley, E. A., Brassel, P. and Moore, D. (1990). A hyaluronan-binding protein shows a partial and temporally regulated codistribution with actin on locomoting chick heart fibroblasts. Exp. Cell. Res. 187, 243-9.
Van Rybroek, J. J. and Olson, M. D. (1981). Surface coat material associated with the cells of the developing lens vesicle in the chick embryo. Anat. Rec. 201, 261-71.

Webster, E. H. and Uknis, E. (1987). Transient appearance of and regional differences in apical cell surface materials during early morphogenesis of the chicken lens. Histochem. J. 19, 203-9.

Wrenn, J. T. and Wessells, N. K. (1969). An ultrastructural study of lens invagination in the mouse. J. Exp. Zool. $171,359-68$.

Wride, M. A. (1996). Cellular and molecular features of lens differentiation: a review of recent advances. Differentiation 61, 77-93.

Yang, J. J. and Hilfer, S. R. (1982). The effect of inhibitors of glycoconjugate synthesis on optic cup formation in the chick embryo. Dev. Biol. 92, 41-53.

Yao, R., Alcala, J. and Maisel, H. (1996). Developmental changes in glyconjugate composition during chick lens morphogenesis. Exp. Eye Res. 62, 419-31.

Yayon, A., Klagsbrun, M., Esko, J. D., Leder, P. and Ornitz, J. D. (1991). Cell surface, heparin-like molecules are required for binding of basic fibroblast growth factor to its high affinity receptor. Cell 64, 841-8.

Yost, H. J. (1990). Inhibition of proteoglycan synthesis eliminates left-right asymetry in Xenopus Laevis cardiac looping. Development 110, 865-74.

Zwaan, J. and Webster, E. H. (1984). Histochemical analysis of extracellular matrix material during embryonic mouse lens morphogenesis in an aphakic strain of mice. Dev. Biol. 104, 380-9. 


\begin{tabular}{|c|c|c|}
\hline & & $\begin{array}{c}\text { AUTHOR QUERIES } \\
\text { Experimental Eye Research (EXER) } \\
\text { Manuscript Number: } 1060 \text { Author(s): Gato et al. }\end{array}$ \\
\hline PAGE & PARA/LINE & QUERY \\
\hline 1 & Col 2-line 2 & Wrenn and Bessells, 1969 - Wessels in refs - [please check?] \\
\hline 3 & Col 1-line 2, & Vector - location please in all cases? \\
\hline 2 & Col 2-line -1 & Sigma - location please? \\
\hline 9 & Ref 23 & Hilfer, 1983 - check volume number? \\
\hline & & \\
\hline & & \\
\hline & & \\
\hline & & \\
\hline & & \\
\hline & & \\
\hline & & \\
\hline & & \\
\hline & & \\
\hline & & \\
\hline & & \\
\hline & & \\
\hline & & \\
\hline & & \\
\hline & & \\
\hline
\end{tabular}

